\title{
Metallurgical Analysis and RF Losses in Superconducting Niobium Thin Film Cavities
}

\author{
D. BLOESS, C. DURAND, E. MAHNER, H. NAKAI ${ }^{\dagger}$ and W. WEINGARTEN \\ CERN - European Organization for Nuclear Research \\ CH - 1211 Geneva 23 (Switzerland) \\ P. BOSLAND \\ C.E. Saclay, F - 91191 Gif-sur-Yvette Cedex (France) \\ J. MAYER \\ MPI für Metallforschung, Seestr. 92, D - 70174 Stuttgart (Germany) \\ L.VANLOYEN \\ IFW, Helmholtzstr. 20, D - 01069 Dresden (Germany)
}

\begin{abstract}
Copper cavities with a thin niobium film - as used in the large electron positron collider LEP - would be also attractive for future linear colliders, provided the decrease of the $Q$-value with the accelerating gradient could be reduced. We aim at extracting the important parameters that govern this decrease. The dependence on the RF frequency is studied by exciting $500 \mathrm{MHz}$ and $1500 \mathrm{MHz}$ cavities in different modes. In addition we combined RF measurements for two $1500 \mathrm{MHz}$ cavities of different $\mathrm{RF}$ performance with microscopic tests (AFM, TEM) on samples cut out of the same cavities. Their micro-structural characterization in plan-view allows to extract the grain size and the defect densities.
\end{abstract}

\section{INTRODUCTION}

Superconducting (sc) accelerating cavities are at the heart of the particle accelerators recently commissioned or upgraded: the recirculating electron linear accelerator CEBAF (presently $4 \mathrm{GeV}$ ) at Thomas Jefferson National Laboratory and the electron positron collider LEP2 (presently $80.5 \mathrm{GeV}$ ) at CERN. The CEBAF accelerator is operated at $1.5 \mathrm{GHz}$ and based on niobium sheet metal cavities, whereas CERN's LEP2 RF system at $352 \mathrm{MHz}$ is (nearly exclusively) built around copper cavities with a thin niobium film inside. This choice is not accidental: niobium sheet metal cavities provide larger accelerating gradients and Q-values above frequencies of, say, $1 \mathrm{GHz}$, whereas thin film cavities give larger Q-values at lower frequencies. However, the Q-value in niobium thin film cavities degrades with the RF field amplitude faster than in niobium sheet cavities.

The aim of this paper is to contribute to the understanding of this degradation - or in other words - why do the RF losses increase more than quadratically with the RF field amplitude (non quadratic losses NQL).

\section{EXPERIMENTAL}

\section{A. Scope of experiment:}

Experimental results on NQL are summarized in [1] and will not be repeated here. Analyzing data from different cavities [2], the frequency dependence of NQL was compatible with both a linear and a quadratic law. Therefore, we measured it again in the same cavity. Secondly, the upper critical magnetic field $B_{\mathrm{c} 2}$ obtained from the trapped magnetic field dependence of the surface resistance was much larger than the one obtained from samples. We therefore cut samples out of two cavities with different RF performance, determined $B_{\mathrm{c} 2}$ again, and several micro-structural parameters.

\section{B. Frequency dependence of $N Q L$ :}

We have partly re-analyzed and partly taken new data on two $500 \mathrm{MHz}$ mono-cell cavities tested in the $\mathrm{TM}_{010}$ and $\mathrm{TM}_{011}$ modes, and on two $1500 \mathrm{MHz}$ mono-cell cavities tested in the $\mathrm{TM}_{010}$ and $\mathrm{TM}_{210}$ modes. These modes were chosen as our fixed coupling probe gave nearly identical coupling to the microwave line after cool down.

A problem to be solved is closely related to the different field distributions of the two modes. One measures the dependence of the Q-value, $Q=\omega U / P \quad(\omega=2 \pi f=$ frequency, $U=$ stored energy, $P=$ dissipated power), on the RF field amplitude, for example the accelerating gradient $E_{\mathrm{a}}$, or the peak magnetic surface field $B_{\mathrm{p}}$. The Qvalue is an average quantity, as it is composed of RF losses at different locations of the cavity surface with different local RF field amplitudes. What one likes to extract is the surface resistance $R_{\mathrm{S}}$ somewhere on the cavity surface as a function of the RF surface magnetic field amplitude $B$ at the same location - tacitly assuming that $R_{\mathrm{S}}$ depends only on $B$ and not on the location. Above all one is interested in the slope $R_{\mathrm{S}}$ ' of $R_{\mathrm{S}}(B)$.

In a cavity excited in the fundamental mode the RF magnetic field $B$ is approximately the same nearly everywhere and identical to its peak value $B_{\mathrm{p}}$. In a good approximation the average surface resistance $\left\langle R_{\mathrm{S}}\right\rangle\left(B_{\mathrm{p}}\right)$ is identical to $R_{\mathrm{S}}(B)$. In a cavity excited in a higher mode, however, this is no longer true. The slope $R_{\mathrm{S}}$ ' is not identical with the one extracted from the $Q\left(B_{\mathrm{p}}\right)$ measurement, a fact which gives rise to a systematic correction (cf. appendix).

Visitor from KEK, 1-1, Oho, Tsukuba, Ibaraki 305 (Japan)

Manuscript received August 27, 1996. 

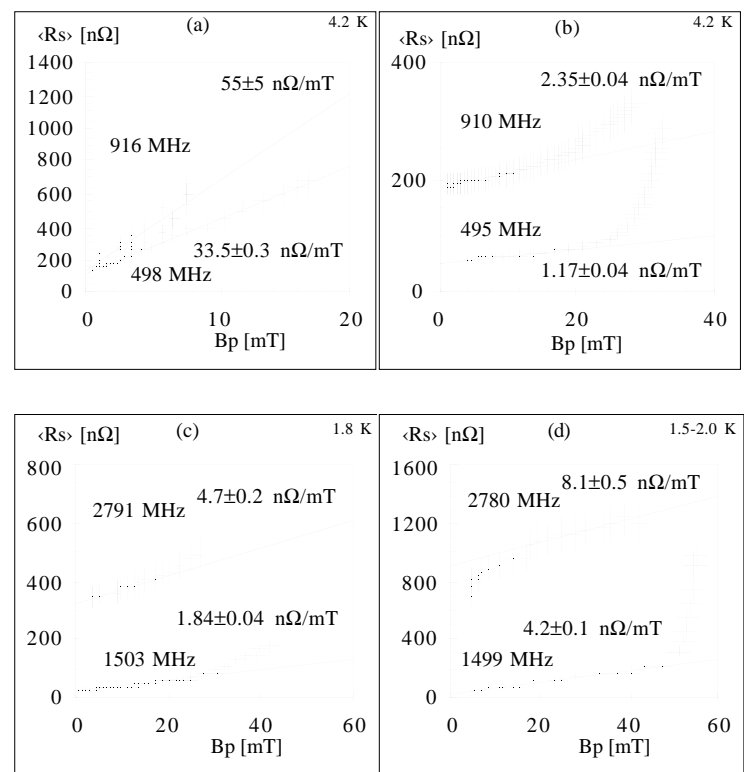

Fig. 1: $\left\langle R_{\mathrm{S}}\right\rangle$ vs. $B_{\mathrm{p}}$ measured for two modes in four different niobium thin film mono-cell cavities (a-d).

Fig. 1 displays the average surface resistance $\left\langle R_{\mathrm{S}}\right\rangle$ vs. the peak RF magnetic surface field amplitude $B_{\mathrm{p}}$ for four different cavities and two different modes. The numbers give the slope $\alpha$ of a linear approximation to the data points. The lower and upper bound of the linear range is chosen such that the error $\Delta \alpha$ is minimized. The ratio $\gamma$ of the slopes $R_{\mathrm{S}}{ }^{\prime}=\alpha / \beta$ (cf. appendix) for the two different modes is compared with their frequency ratio and its square (Table I). The error in $\gamma$ comprises the errors $\Delta \alpha$ and $\Delta \beta$. The latter is based on the computer simulation of the field distribution in a cavity. Results from different codes (URMEL and SUPERFISH [3]) deviated by $7 \%$. We therefore estimated the error $\Delta \beta= \pm 14 \%$.

TABLE I

RATio $\gamma$ Of $R_{\mathrm{S}}$ ' For DifFerent Modes

\begin{tabular}{cccccc}
\hline Cavity & $\begin{array}{c}f_{1} \\
{[\mathrm{MHz}]}\end{array}$ & $\begin{array}{c}f_{2} \\
{[\mathrm{MHz}]}\end{array}$ & $f_{2} / f_{1}$ & $\left(f_{2} / f_{1}\right)^{2}$ & $\begin{array}{c}\text { Ratio of } \\
\text { slopes } \gamma\end{array}$ \\
\hline a & 498 & 916 & 1.8 & 3.4 & $1.9 \pm 0.4$ \\
b & 495 & 910 & 1.8 & 3.4 & $2.3 \pm 0.4$ \\
c & 1503 & 2791 & 1.9 & 3.4 & $4.2 \pm 0.9$ \\
d & 1499 & 2780 & 1.9 & 3.4 & $3.2 \pm 0.7$ \\
\hline
\end{tabular}

C. Analysis of samples cut from cavities:

Samples were cut from two $1500 \mathrm{MHz}$ niobium film cavities (we call them "as received"), the RF results of which were quite different (Fig. 2 and Table II). They are taken from ref. 4, except for the slope $\alpha$. It is determined as described before to facilitate the comparison.

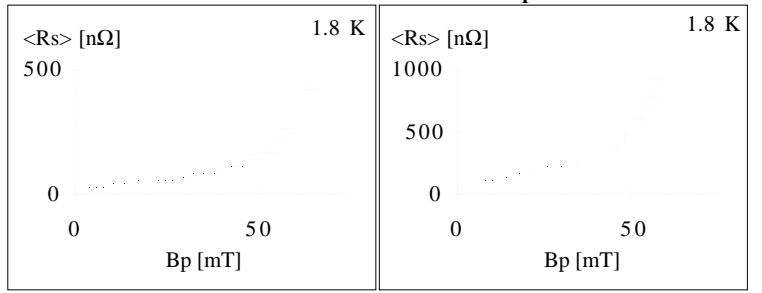

Fig. 2: $\left\langle R_{c}\right\rangle$ vs. $B_{n}$ for two $1500 \mathrm{MHz}$ niohium film cavities
TABLE II

COMPARISON OF RF PERformance OF CAVITIES \#1 AND \#2 (UPPER PART) AND METAlluRgy OF THE CORRESPONDING SAMPLES (LOWER PART)

\begin{tabular}{|c|c|c|}
\hline Physical quantity & Cavity \#1 & Cavity \#2 \\
\hline BCS surface resistance $[\mathrm{n} \Omega], 4.2 \mathrm{~K}$ & $405 \pm 11$ & $400 \pm 15$ \\
\hline Residual surface resistance $R_{\text {res }}[\mathrm{n} \Omega]$ & $32 \pm 5$ & $75 \pm 5$ \\
\hline Critical temperature $T_{\mathrm{c}}[\mathrm{K}]$ & $9.506 \pm 0.016$ & 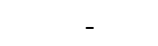 \\
\hline Slope $R_{\mathrm{S}}{ }^{\prime}[\mathrm{n} \Omega / \mathrm{mT}], 1.8 \mathrm{~K}$ & $1.2 \pm 0.1$ & $6.3 \pm 0.3$ \\
\hline Penetration depth $\lambda_{0}[\mathrm{~nm}]^{\text {a) }}$ & $82 \pm 3$ & - \\
\hline Critical temperature $T_{\mathrm{c}}[\mathrm{K}]^{\mathrm{b}}$ & $9.61-9.79$ & $9.35-21.74$ \\
\hline (after immersion in $\mathrm{HNO}_{3}$ ) & $9.16-9.21$ & $9.15-9.19$ \\
\hline Upper critical field $B_{\mathrm{c} 2}[\mathrm{~T}], 4.2 \mathrm{~K}$ & $1.50 \pm 0.05$ & $2.6 \pm 0.1$ \\
\hline (after immersion in $\mathrm{HNO}_{3}$ ) & $0.84 \pm 0.02$ & $0.80 \pm 0.02$ \\
\hline "Structure" length ${ }^{c)}[\mathrm{nm}](\mathrm{AFM})$ & 200 & 100 \\
\hline Roughness $R_{\mathrm{a}}[\mathrm{nm}](\mathrm{AFM})$ & 3 & 3 \\
\hline Grain size $[\mathrm{nm}]$ (TEM) & $100-500$ & $50-200$ \\
\hline Defect density $\left[\mathrm{nm}^{-3}\right]$ (TEM) & $10^{-4}$ & $10^{-3}$ \\
\hline$R R R(10 \mathrm{~K})$ & $13.0 \pm 0.5$ & $9.0 \pm 0.5$ \\
\hline Film thickness $[\mu \mathrm{m}]$ & 3 & 3 \\
\hline
\end{tabular}

1) The upper critical field $B_{c 2}$ and the critical temperature $T_{\mathrm{c}}$ : $B_{\mathrm{c} 2}$ was measured at $4.2 \mathrm{~K}$ by two coils with coplanar windings and the sample in between. The whole set-up is exposed to a static magnetic field ( $8 \mathrm{~T} \mathrm{sc}$ magnet) directed parallel to the sample's surface. When the primary coil is fed with a small AC current, the pickup signal in the secondary coil depends on that part of the sample surface which cannot be encircled by a closed sc path. The cleanest signal (Fig. 3) was obtained for a niobium film on a copper substrate (thinned down to $1 \mathrm{~mm}$ ), an AC magnetic field of $50 \mathrm{mG}$ at $10 \mathrm{~Hz}$. The critical temperature $T_{\mathrm{c}}$ was measured with the same apparatus at vanishing static magnetic field by slowly warming up the sample.
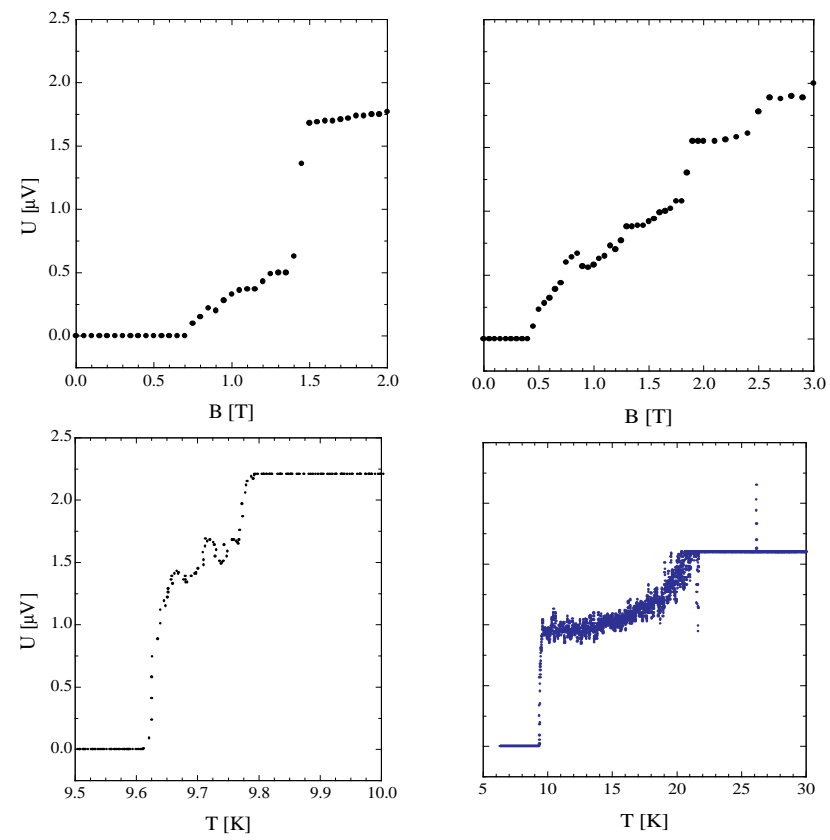

Fig. 3: Pickup signal for samples \#1 (left) and sample \#2 (right) vs. the static magnetic field (top row) and vs. the temperature

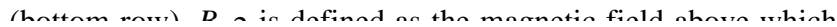


For a similar set of samples the copper substrate was removed by immersion in diluted nitric acid. The results changed markedly (Fig. 4). $B_{\mathrm{c} 2}$ and $T_{\mathrm{c}}$ were the same for the two samples and much closer to the values of bulk niobium.
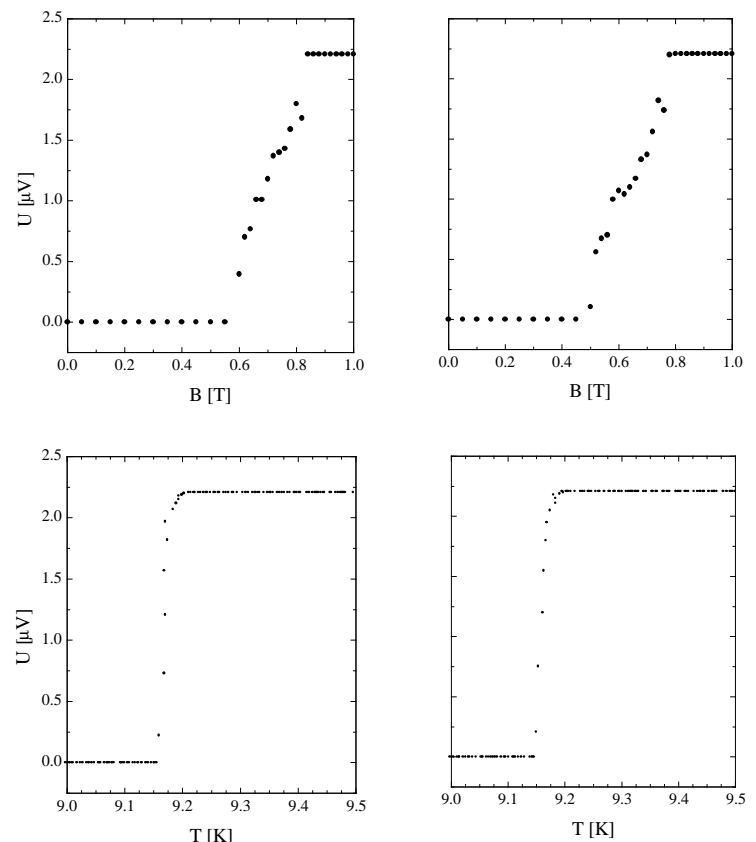

Fig. 4: Same as Fig. 3 for similar samples after immersion in nitric acid to remove the copper substrate.

2) Measurements with an Atomic Force Microscope (AFM) and a Transmission Electron Microscope (TEM):
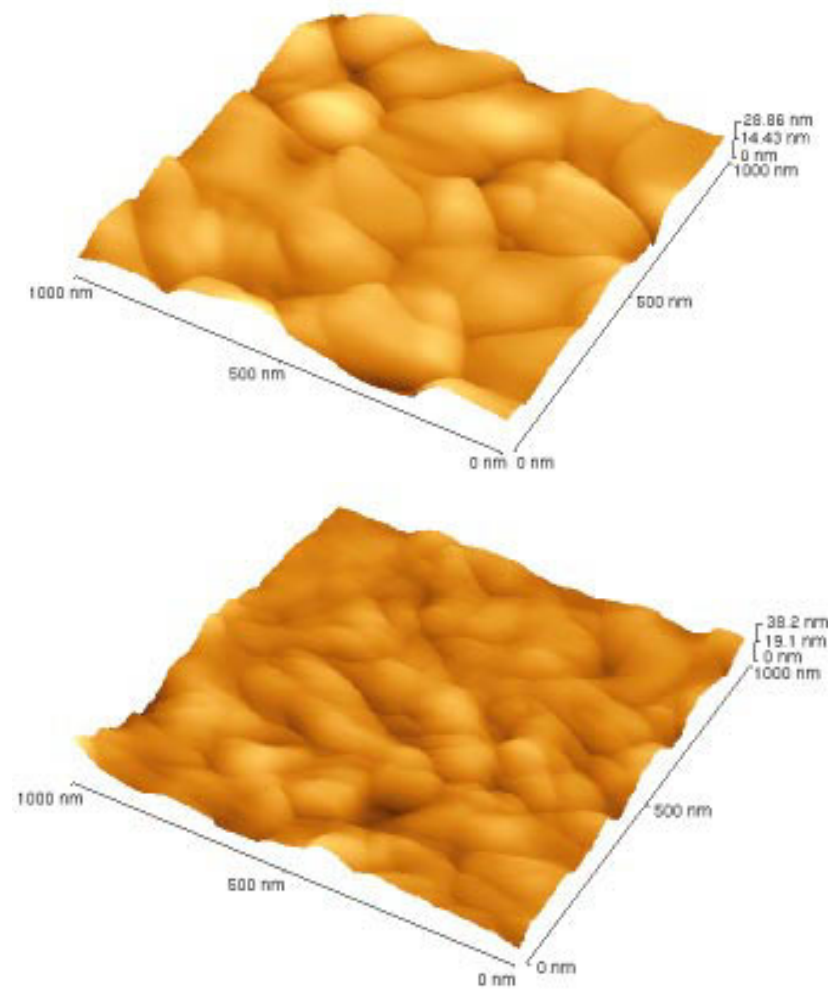

Fig. 5: AFM pictures of samples \#1 (top) and \#2 (bottom). We call the average distance of the grooves "structure length" to distinguish it from the grain size. It turns out that it is equal to the
The AFM measurements were performed with the Explorer AFM of TopoMetrix at IFW Dresden, the TEM analysis was done with the Zeiss EM 912 Omega at MPI Stuttgart.

The image resolution of the AFM pictures is $300 \mathrm{X} 300$ pixels. The AFM pictures show a "structure" with a characteristic length of about $200 \mathrm{~nm}$ in sample \#1, about twice the one of sample \#2 (Fig. 5). This length can be interpreted as a typical grain size. The samples have about similar average roughness $\left(R_{\mathrm{a}}=3 \mathrm{~nm}\right)$.

The TEM measurements were performed on samples which were cut parallel (plan view) and perpendicular (cross-section) to the niobium film plane. Standard preparation techniques involving mechanical polishing and ion beam thinning the specimens revealed a columnar grain growth perpendicular to the interface. The rodshaped like $\mathrm{Nb}$ grains reach dimensions of up to several $\mu \mathrm{m}$ in length, which is basically the deposited film thickness. The diameter of the columnar grains can best be studied in the plan view specimens. Typical micrographs for cavities \#1 and \#2 are shown in Fig. 6 together with a reference sample made from bulk niobium. The typical grain sizes in the plane parallel to the film are $100-500$ $\mathrm{nm}$ for sample \#1 and 50 - $200 \mathrm{~nm}$ for sample \#2. From the micrographs a high density of defects is also evident. To ensure that the defects are not introduced during specimen preparation we have also studied the bulk $\mathrm{Nb}$ sample which was prepared in exactly the same way. The defects presumably consist of dislocations, dislocation loops and point defect agglomerates. Mean defect densities per $\mathrm{nm}^{3}$ were estimated to be $10^{-4}$ for sample \#1 and $10^{-3}$ for sample \#2. These values were obtained by measuring mean distances between defects in several micrographs and are based on an estimated specimen thickness of 20 $30 \mathrm{~nm}$. The defect density in the bulk $\mathrm{Nb}$ reference sample was lower by about three orders of magnitude confirming that specimen preparation is not a source of the high defect densities measured for the samples from the cavities.
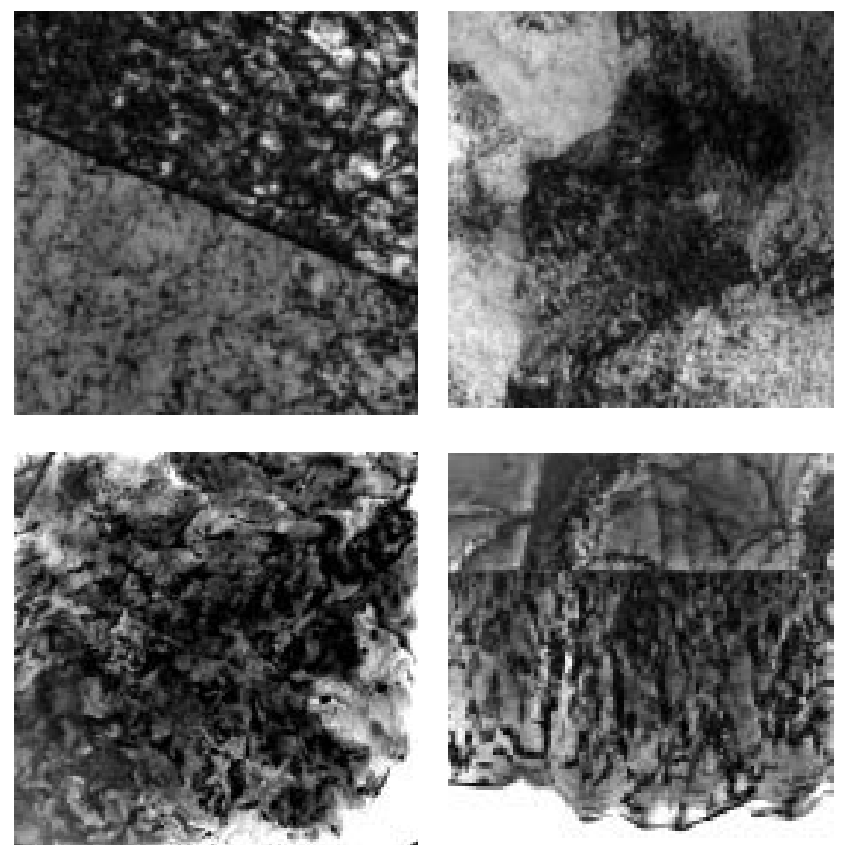

Fig. 6: TEM pictures (from top left to bottom right) in plan view of hulk nishium reference camnle $(R R R=20 \cap)$ camnle $\# 1$ 
3) Measurements with a Scanning Electron Microscope (SEM) and a Light Microscope (LM): All pictures (Figs. 7 and 8) display etching patterns from the copper substrate. There are coarse and fine structures visible, which are similar for the samples \#1 and \#2. The SEM pictures suggest the presence of holes, which is not confirmed for a larger magnification yet.
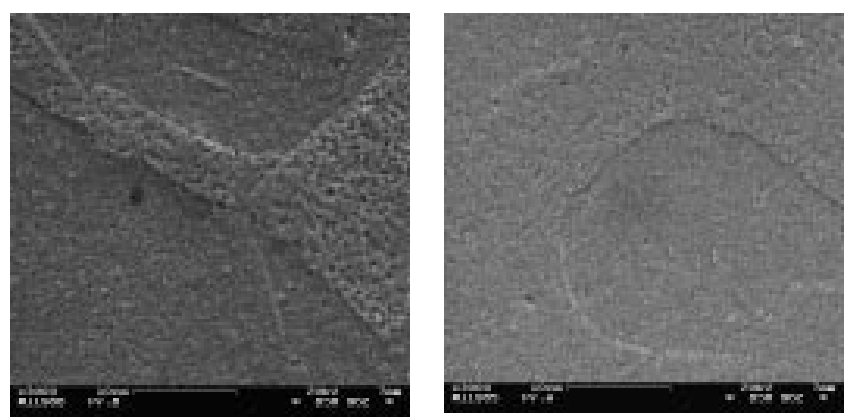

Fig. 7: SEM pictures of sample \#1 (left) and sample \#2 (right). The image width is $40 \mu \mathrm{m}$.
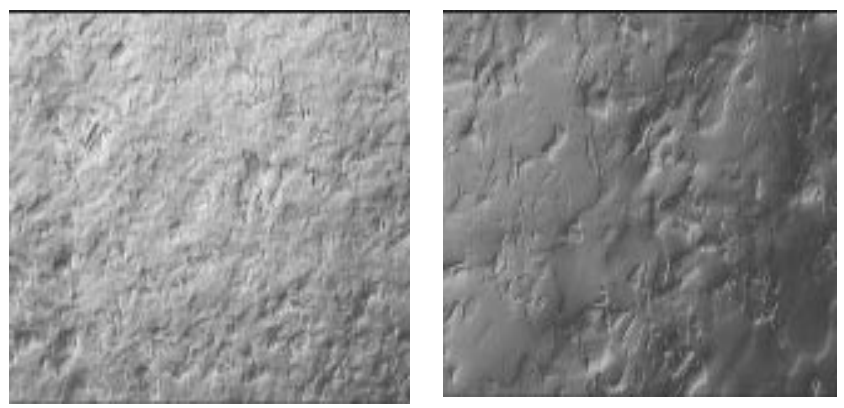

Fig. 8: LM pictures of sample \#1 (left) and sample \#2 (right). The image width is $1 \mathrm{~mm}$.

\section{DISCUSSION}

The data are compatible with a linear dependence of the surface resistance on frequency below $1.5 \mathrm{GHz}$ (Table I). This fact confirms earlier data obtained on a series of $350 \mathrm{MHz}$ and $1500 \mathrm{MHz}$ cavities [1]. In addition, the data suggest a quadratic dependence above $1.5 \mathrm{GHz}$, which may signify that near a crossover frequency between 1.5 and $3 \mathrm{GHz}$ another loss mechanism becomes dominant. How can it be understood?

The analytic expression for NQL [2] is (besides numerical factors near unity) equivalent to that of $\mathrm{AC}$ losses in a superconductor (Bean model). They are described in linear approximation in $B$ by the surface resistance (with the critical current density $j_{\mathrm{c}}$ of Bean's formula [5] replaced by $\left.B_{\mathrm{c} 2} /\left(\mu_{0} l\right)\right)$,

$$
R_{s}=\frac{2}{3 \pi} \omega \mu_{0} l \underbrace{l \frac{B}{B_{c 2}(T)}}_{f(T, B)} .
$$

$l$ is the depth of the surface layer, which contributes to NQL. $R_{\mathrm{S}}$ is proportional to the fraction $f$ of metal becoming normal-conducting (nc) with increasing $B$. The precise analytic form of $f$ is not known. $f$ depends on the temperature and is here approximated by the ratio of the RF magnetic field $B$ and the upper critical magnetic field $B_{\mathrm{c} 2}$ of the film, $f(T, B)=B / B_{\mathrm{c} 2}(T)$. A linear increase of the surface resistance with frequency reflects the fact that loss mechanism must appear. It has the same physical basis as the BCS losses and hence increases with the square of the frequency. It rises from the fact that a thin nc surface layer or grain boundaries [6] feel the voltage from the inductance of the sc electrons. In the two fluid model, for a surface fraction $f(T)$ of nc electrons, the corresponding surface resistance for sufficiently low temperatures $\left(T<T_{\mathrm{C}} / 2\right)$ is

$$
R_{s}=\frac{1}{2} \mu_{0}^{2} \sigma_{n} \lambda_{0}^{3} \omega^{2} f(T)
$$

$\left(\sigma_{\mathrm{n}}=\right.$ conductivity at $10 \mathrm{~K}, \lambda_{0}=$ penetration depth, $f(T)=$ $\left.\left(T / T_{\mathrm{c}}\right)^{4}\right)$ [7]. Therefore, above some frequency $\omega_{\mathrm{c}}$, the RF losses described by (2) exceed those described by (1).

By equating (1) and (2), and the replacement $f(T) \rightarrow f(T$, $B$ ) in (2), one obtains for the depth $l$ of the lossy surface layer

$$
l=\frac{3 \pi}{4} \mu_{0} \sigma_{n} \lambda_{0}^{3} \omega_{\mathrm{c}} .
$$

With a typical conductivity for niobium at room temperature $\left(7.6 \cdot 10^{6}(\Omega \mathrm{m})^{-1}\right)$ and $R R R=10$, one obtains a conductivity of $\sigma_{\mathrm{n}}=0.8 \cdot 10^{8}(\Omega \mathrm{m})^{-1}$ at $10 \mathrm{~K}$. By inserting a crossover frequency close to the observed value, $\omega_{\mathrm{c}}=$ $2 \pi \cdot 3 \cdot 10^{9} \mathrm{~s}^{-1}, \lambda_{0}=85 \mathrm{~nm}$ [8], one finds for the depth $l=3$ $\mathrm{nm}$. We point out that this number gives only the order of magnitude. It implies that the sensitive region is confined to the uppermost surface layer. This is a reassuring consequence, because it is compatible with the observation that the NQL are sensitive to the surface [9].

The "as received" sample \#1 (good RF performance) has a lower $B_{\mathrm{c} 2}$ with a sharper transition, a smaller transition width in $T_{\mathrm{c}}$, a factor of two larger structure length (AFM), a larger grain size (TEM), a larger $R R R$ value and one order of magnitude lower defect density, compared to sample \#2 (poor RF performance). $R_{\mathrm{BCS}}$ and the niobium average surface roughness are quite similar.

Immersion in nitric acid not only removes the copper substrate, but also oxidizes the niobium surface. After applying this treatment, the two samples \#1 and \#2 become very similar with respect to $B_{\mathrm{c} 2}$ and $T_{\mathrm{c}}$. Therefore, the discrepancy in $B_{\mathrm{c} 2}$ and $T_{\mathrm{c}}$ observed in the "as received" samples \#1 and \#2 could again be a surface effect. But one cannot exclude an effect of the $\mathrm{NbCu}$ interface.

These observations may be explained by a common feature, confirming previous results [9]: the uppermost film surface is a mixture of phases containing extreme type II niobium with a very short coherence length ("disordered niobium" according to R. Vaglio [10]). A deeper discussion has to be presented elsewhere.

\section{CONCLUSION}

The frequency dependence of $R_{\mathrm{S}}$ ' allows an estimate of the depth $l$ of the surface layer giving rise to NQL (several $\mathrm{nm}$ ). The comparative measurements on samples cut from cavities with different RF performance are in accordance with the following explanation.

To the authors' best knowledge the features observed in niobium thin film cavities - as summarized in ref. 1 - can be explained as follows: the film surface has a coherence length, which spans the interval from that of bulk niobium down to several $\mathrm{nm}$. The NQL appear when the RF 
the niobium surface). They are induced in a - compared to the penetration depth - thin surface layer by the gradual destruction and re-establishment of superconductivity (or equivalently entry and exit of magnetic flux) within one RF half cycle and the dissipation into the lattice of the heat involved. They are described by (1) for frequencies below about $1 \mathrm{GHz}$ and for $B>B_{\mathrm{c} 1}$. As an example, with $f=352$ $\mathrm{MHz}, l=3 \mathrm{~nm}, B_{\mathrm{c} 2}=1.5 \mathrm{~T}, T=4.2 \mathrm{~K}$, one obtains $R_{\mathrm{S}}{ }^{\prime}=$ $1.2 \mathrm{n} \Omega / \mathrm{mT}$ for the LEP cavities, which is the observed value [1].

\section{APPENDIX}

The geometry factor $G$ is defined as

$$
G=\omega \mu_{0} \frac{\int d v B^{2}}{\oint d a B^{2}},
$$

and the Q-value (as a function of the peak surface magnetic field amplitude $\left.B_{\mathrm{p}}\right)$ is defined as

$$
Q\left(B_{p}\right)=\omega \frac{\int d \mathrm{v} B^{2}\left(B_{p}\right)}{\oint d \mathrm{a} R_{\mathrm{s}} B^{2}\left(B_{p}\right)},
$$

where the two integrals are taken over the volume $V$ and the surface $A$ of the cavity. The average surface resistance $\left\langle R_{\mathrm{S}}\right\rangle$ is defined as

$$
\left\langle R_{s}\right\rangle\left(B_{p}\right)=\frac{G}{Q\left(B_{p}\right)} .
$$

With a parametrisation of the local surface resistance $R_{\mathrm{S}}$ as a function of the local surface magnetic field amplitude $B$,

one finds

$$
R_{S}(B)=R_{s 0}+R_{s}^{\prime} \cdot B+\ldots
$$

$$
\left\langle R_{s}\right\rangle\left(B_{p}\right)=R_{s 0}+R_{s}^{\prime} \underbrace{\frac{\oint d \mathrm{a} B^{3}\left(B_{p}\right)}{B_{p} \oint d \mathrm{a} B^{2}\left(B_{p}\right)}}_{\beta} \cdot B_{p}+\ldots
$$

which is abbreviated as

$$
\left\langle R_{s}\right\rangle\left(B_{p}\right)=R_{s 0}+\alpha \cdot B_{p}+\ldots .
$$

The slope $\alpha$ of the average surface resistance $\left\langle R_{\mathrm{S}}\right\rangle$ is linked with the slope $R_{\mathrm{S}}$ ' of the local surface resistance $R_{\mathrm{S}}$ as

$$
R_{s}^{\prime}=\frac{\alpha}{\beta} .
$$

The $\beta$ factors were computed with URMEL and are summarized in Table III.

TABLE III

CORRECTION FACTOR FOR SLOPE $R_{\mathrm{S}}$ '

\begin{tabular}{cc}
\hline Mode (Frequency [MHz]) & $\beta$ \\
\hline $\mathrm{TM}_{010}(500)$ & 0.96 \\
$\mathrm{TM}_{011}(910)$ & 0.85 \\
$\mathrm{TM}_{010}(1500)$ & 0.93 \\
$\mathrm{TM}_{210}(2790)$ & 0.56 \\
\hline
\end{tabular}

well as the preparation of several samples were performed by R. Russo, whom we thank gratefully.

\section{REFERENCES}

[1] W.Weingarten,Proc.7thWorkshopRFSuperconductivity, Saclay, France, 17 - 20 Oct. 1995, ed. B. Bonin, Gif-sur-Yvette 1996, p. 129, to be published in Part. Acc..

[2] C. Durand et al., IEEE Trans. Appl. Supercond. 5 (1995) 1107.

[3] URMEL : T. Weiland, DESY 82-015 and DESY M-82-24. SUPERFISH: K. Halbach and R. F. Holsinger, Part. Acc. 7 (1976) 213.

[4] P. Darriulat et al., ibid. ref. 1, 467.

[5] Cf. e.g. A. M. Portis, Electrodynamics of High Temperature Superconductors, Singapore 1993, p. 85.

[6] J. P. Carini et al., Phys. Rev. B37 (1988) 9726.

[7] Cf. e.g. J. H. Hinken, Superconductor Electronics, Berlin, Heidelberg 1989 , p. 22

[8] R. J. Donnelly, "Cryogenics", in Physics Vade Mecum, ed. H. L. Anderson, AIP 1981.

[9] Ph. Bernard et al., Proc. 3rd EPAC Berlin, March 24 - 28, 1992, eds. H. Henke, H. Homeyer, Ch. Petit-Jean-Genaz, Gif-sur-Yvette 1992, p. 1269.

[10] C. Camerlingo et al., Phys. Rev. B31 (1985) 3121.

\section{ACKNOWLEDGMENTS}

We thank D. Boussard for his comments when reading 